\title{
Predictors of anaemia and iron deficiency in HIV-infected pregnant women in Tanzania: a potential role for vitamin D and parasitic infections
}

\author{
Julia L Finkelstein ${ }^{1,2,3, *}$, Saurabh Mehta ${ }^{1}$, Christopher P Duggan ${ }^{2,4}$, Donna \\ Spiegelman ${ }^{3,5}$, Said Aboud ${ }^{6}$, Roland Kupka ${ }^{2,7}$, Gernard I Msamanga ${ }^{8}$ and \\ Wafaie W Fawzi ${ }^{2,3,9}$ \\ 'Division of Nutritional Sciences, Cornell University, Ithaca, NY 14853, USA: ${ }^{2}$ Department of Nutrition, \\ Harvard School of Public Health, Boston, MA, USA: ${ }^{3}$ Department of Epidemiology, Harvard School of Public \\ Health, Boston, MA, USA: ${ }^{4}$ Division of GI/Nutrition, Children's Hospital Boston, MA, USA: ${ }^{5}$ Department of \\ Biostatistics, Harvard School of Public Health, Boston, MA, USA: ${ }^{6}$ Department of Microbiology and Immunology, \\ Muhimbili University of Health and Allied Sciences, Dar es Salaam, Tanzania: ${ }^{7}$ United Nations Children's Fund, \\ Regional Office for West and Central Africa, Dakar, Senegal: ${ }^{8}$ Department of Community Health, Muhimbili \\ University of Health and Allied Sciences, Dar es Salaam, Tanzania: ${ }^{9}$ Department of Global Health and \\ Population, Harvard School of Public Health, Boston, MA, USA
}

Submitted 5 December 2010: Accepted 23 August 2011: First published online 4 October 2011

\begin{abstract}
Objective: Anaemia is common during pregnancy, and prenatal Fe supplementation is the standard of care. However, the persistence of anaemia despite Fe supplementation, particularly in HIV infection, suggests that its aetiology may be more complex and warrants further investigation. The present study was conducted to examine predictors of incident haematological outcomes in HIV-infected pregnant women in Tanzania.

Design: Prospective cohort study. Cox proportional hazards and binomial regression models were used to identify predictors of incident haematological outcomes: anaemia $(\mathrm{Hb}<110 \mathrm{~g} / \mathrm{l})$, severe anaemia $(\mathrm{Hb}<85 \mathrm{~g} / \mathrm{l})$ and hypochromic microcytosis, during the follow-up period.

Setting: Antenatal clinics in Dar es Salaam, Tanzania.

Subjects: Participants were 904 HIV-infected pregnant women enrolled in a randomized trial of vitamins (1995-1997).

Results: Malaria, pathogenic protozoan and hookworm infections at baseline were associated with a two-fold increase in the risk of anaemia and hypochromic microcytosis during follow-up. Higher baseline erythrocyte sedimentation rate and CD8 T-cell concentrations, and lower $\mathrm{Hb}$ concentrations and CD4 T-cell counts, were independent predictors of incident anaemia and Fe deficiency. Low baseline vitamin $\mathrm{D}(<32 \mathrm{ng} / \mathrm{ml})$ concentrations predicted a 1.4 and 2.3 times greater risk of severe anaemia and hypochromic microcytosis, respectively, during the follow-up period. Conclusions: Parasitic infections, vitamin D insufficiency, low CD4 T-cell count and high erythrocyte sedimentation rate were the main predictors of anaemia and Fe deficiency in pregnancy and the postpartum period in this population. A comprehensive approach to prevent and manage anaemia, including micronutrient supplementation and infectious disease control, is warranted in HIV-infected women in resource-limited settings - particularly during the pre- and postpartum periods.
\end{abstract}

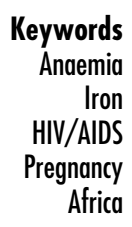

An estimated 1.6 billion people are anaemic worldwide, and anaemia is common during pregnancy ${ }^{(1)}$. Approximately $50 \%$ of pregnant women have anaemia $(\mathrm{Hb}<110 \mathrm{~g} / \mathrm{l})$ in resource-limited settings, compared with $12-25 \%$ in developed regions ${ }^{(1)}$. Several studies in Sub-Saharan Africa have identified a prevalence of $80 \%$ or higher in HIV-infected pregnant women ${ }^{(2-5)}$.
Fe deficiency is the leading cause of anaemia worldwide and in pregnancy ${ }^{(6)}$. The consequences of anaemia and $\mathrm{Fe}$ deficiency in pregnancy have been well established ${ }^{(7)}$, and include maternal and infant mortality ${ }^{(8)}$ and low birth weight ${ }^{(9)}$. A substantial body of evidence also supports the relationships between Fe deficiency and poorer cognitive development in children ${ }^{(10)}$ and reduced 
work capacity in adults ${ }^{(11)}$. Fe supplementation (with folic acid) is standard prenatal care in most countries, based on its likely benefits in preventing maternal anaemia and related complications ${ }^{(12)}$.

The aetiology of anaemia in the context of HIV is particularly complex. In addition to Fe deficiency, anaemia of inflammation is a leading cause of anaemia in HIVinfected individuals ${ }^{(13)}$. Nutritional deficiencies of folate and vitamin $\mathrm{B}_{12}{ }^{(14)}$ and concurrent infections may also contribute to the risk of anaemia. Studies in HIV-infected pregnant women have found an extremely high prevalence of anaemia, despite presumed availability of $\mathrm{Fe}$ supplementation $^{(2-5)}$; however, most have focused on cross-sectional assessments of anaemia prevalence during pregnancy. Risk factors for incident haematological outcomes need to be examined, to elucidate the aetiology of anaemia in HIV-infected pregnant women receiving $\mathrm{Fe}$ supplementation.

We conducted a prospective observational analysis of incident anaemia and Fe deficiency during pregnancy and the postpartum period in HIV-infected women who were pregnant at enrolment and followed throughout the postpartum period.

\section{Methods}

\section{Study design}

Participants were pregnant women between 12 and 27 weeks of gestation who were enrolled in the Trial of Vitamins (TOV), a double-blind, placebo-controlled randomized trial conducted in Dar es Salaam, Tanzania (1995-1997). That study was conducted to examine the effects of daily micronutrient supplementation to HIVinfected pregnant women on the risks of mother-to-child HIV transmission, HIV disease progression and adverse perinatal outcomes. The detailed design of the trial has been described previously ${ }^{(15)}$.

All women received $120 \mathrm{mg}$ of ferrous Fe (as ferrous sulfate) and $5 \mathrm{mg}$ of folate daily during pregnancy starting at their first antenatal clinic visit, and chloroquine $(300 \mathrm{mg})$ weekly as malaria prophylaxis, as per the then current standard of care in Tanzania. Women received bottles of ninety tablets of Fe-folate supplements and were followed up at monthly visits. HIV/AIDS care was provided according to WHO guidelines; antiretroviral therapy (ART) was not available to most women in Tanzania at the time of the study, including participants in this trial.

\section{Etbics}

Informed consent was obtained from all participants. The research protocol was approved by the Research and Publications Committee of Muhimbili University College of Health Sciences, the Ethical Committee of the Tanzanian National AIDS Control Program and the Institutional Review Board of the Harvard School of Public Health.

\section{Assessment of baseline covariates}

Structured interviews were conducted at the initial clinic visit (i.e. 12-27 weeks' gestation, referred to as 'baseline'), to collect information on demographic characteristics, including age, educational level and socio-economic status, symptoms and obstetric history. Gestational age was determined based on the date of the last menstrual period and assessment by a trained nurse. Physicians conducted a medical evaluation of HIV-related symptoms, disease stage and clinical morbidities; HIV disease stage was determined based on WHO guidelines ${ }^{(16)}$. Blood, stool, urine and vaginal swab specimens were collected at enrolment to assess co-infections, including sexually transmitted infections, malaria and helminth infections. Anthropometric measurements, including height, weight and mid-upper arm circumference, were obtained using standardized procedures and calibrated instruments.

\section{Follow-up}

Of the 1078 women in the trial, three were not pregnant, six died before delivery and one was WHO HIV disease stage IV. Nine hundred and four women had a baseline $\mathrm{Hb}$ measurement and at least one measurement thereafter, and were included in the present analyses (Fig. 1). Women were followed for a median of 57 months (interquartile range (IQR): 29-67 months), with a mean of 8.6 (sD 4) Hb measurements (median: 9, IQR: 5-12).

Clinical evaluations were performed monthly to assess HIV-related complications, disease stage and clinical morbidities $^{(16)}$. Women who missed a clinic visit or travelled outside Dar es Salaam were followed-up via home visits.

\section{Laboratory metbods}

Whole blood samples were collected from participants at baseline, delivery, 6 weeks postpartum and every 6 months thereafter. Laboratory samples were tested in batch, and instruments were calibrated daily using standardized procedures.

HIV serostatus was determined by Enzygnost antiHIV-1/2 Plus (Dade Behring, Marburg, Germany) followed by the Wellcozyme HIV-1 recombinant test (Murex Biotech Ltd, Dartford, UK). Discordant ELISA results were resolved by Western blot (Bio-Rad Laboratories Ltd, Hertfordshire, UK) assay ${ }^{(17)}$.

$\mathrm{Hb}$ concentrations were assessed using a CBC5 Coulter Counter (Coulter Corporation, Miami, FL, USA) or the cyanmethaemoglobin method with a colorimeter (Corning Inc., Corning, NY, USA). Thin blood films with Leishman's stain were prepared and examined microscopically. Hypochromasia, microcytosis and macrocytosis were classified into four levels, coded as absent, $1+, 2+$ or $3+$.

Total leucocyte counts were evaluated with a CBC5 Coulter Counter (Coulter Corporation). Absolute CD3, CD 4 and CD8 T-cell counts were determined with the FACSCount system (Becton-Dickinson, San Jose, CA, USA); differential white blood cell counts were evaluated manually. 
A complete blood count was obtained (Coulter Corporation), and erythrocyte sedimentation rate (ESR) was determined.

Measurements of infections, including malaria, intestinal parasites and genital infections, were conducted in all participants only at the baseline clinic visit. Additionally, if a participant presented with symptoms suggestive of malaria or other infections, they were treated as per the clinical guidelines of WHO and the Ministry of Health of Tanzania at that time. Malaria parasites were identified in thick-smear blood films stained with Giemsa. Malaria parasite density was calculated based on a leucocyte count of $8000 \times 10^{6} / 1$ blood $^{(18)}$. Urine samples were examined for the presence of Schistosoma haematobium. Sera and genital swabs were tested for vaginal candidiasis and sexually transmitted infections including syphilis, gonorrhoea and trichomoniasis. To identify intestinal helminths (hookworm, Ascaris lumbricoides, Trichuris trichura, Strongyloides stercoralis and Schistosoma mansoni) and pathogenic protozoan infections (Giardia lamblia, Entamoeba bistolytica and Cryptosporidium parvum), stool specimens were first examined macroscopically for general characteristics (pus, mucus, blood) and worms. Stools were then examined microscopically using saline wet mount for detection of eggs, larvae protozoan trophozoites and cysts, followed by iodine wet mount to identify cysts. The formalin-ether concentration technique was used for further identification of eggs, larvae and cysts. Co-infections were treated at the time of diagnosis, in accordance with clinical guidelines of the Ministry of Health of Tanzania.

Serum 25-hydroxy vitamin D (25(OH)D) concentrations were measured using the fully automated chemiluminescence ADVANTAGE 25(OH)D assay (Nicholas Institute Diagnostics, San Juan Capistrano, CA, USA). Vitamin D insufficiency was dichotomized at $32 \mathrm{ng} / \mathrm{ml}$, based on requirements for optimal Ca homeostasis ${ }^{(19)}$ and in accordance with previous studies in this trial ${ }^{(20)}$. $25(\mathrm{OH}) \mathrm{D}$ concentrations were measured in all participants, using blood samples collected at the baseline clinic visit.

\section{Assessment and definitions of outcomes}

Anaemia and severe anaemia were defined as $\mathrm{Hb}$ less than $110 \mathrm{~g} / 1$ and $85 \mathrm{~g} / 1$, respectively, in accordance with WHO criteria and clinical guidelines in Tanzania. We examined the morphology of peripheral erythrocytes as a proxy to identify Fe deficiency (presence of hypochromic and microcytic cells) and vitamin $\mathrm{B}_{12}$ and folate deficiency (presence of macrocytic cells) $^{(21)}$. Hypochromic microcytic anaemia was categorized as severe (hypochromasia $\geq 2+$ and microcytic cells observed), moderate and above (hypochromasia $\geq 1+$ and microcytic cells observed), and mild and above (hypochromasia $\geq 1+$ with or without microcytosis). Participants diagnosed with severe anaemia received clinical management as per standard of care, including Fe supplementation.

\section{Statistical analyses}

We used Cox proportional hazard models to examine predictors of time to categorical haematological endpoints: anaemia, severe anaemia and hypochromic microcytosis ${ }^{(22)}$. For each analysis, we examined time to the first occurrence of each haematological endpoint during follow-up; women who had the endpoint of interest at baseline were excluded. For those without the outcomes, follow-up ended on the date on which HIV disease stage was last assessed. We also investigated predictors of resolution of haematological endpoints during pregnancy, by conducting binomial regression analyses among individuals with the outcome of interest at baseline, to examine if these endpoints resolved at delivery.

\section{Covariates}

Conventional cut-offs were used to categorize risk factors, where available; otherwise, medians were used to classify variables, as is consistent with previous publications from the $\mathrm{TOV}^{(2,23)}$. BMI was categorized as $<18 \cdot 5,18 \cdot 5-<25 \cdot 0$, $25 \cdot 0-<30 \cdot 0$ and $\geq 30 \cdot 0 \mathrm{~kg} / \mathrm{m}^{2(24)}$. CD 4 T-cell counts were categorized as $<200,200-499$ or $\geq 500$ cells $/ \mu l$, and CD3 and CD8 T-cell counts were evaluated based on their median concentrations. Malaria parasitaemia was categorized as light $(1-999 / \mu \mathrm{l})$, moderate $(1000-9999 / \mu \mathrm{l})$ or heavy $(\geq 10000 / \mu \mathrm{l})$. Intestinal parasites were categorized by the presence of ova or larvae in stool specimens. Presumed adherence to prenatal Fe supplements was defined as the number of days for which supplements were available divided by the total number of days between enrolment and delivery, and was included as a covariate in all multivariate analyses.

Variables with univariate $P$ values of less than $0 \cdot 20$ were included in each of the multivariate regression models and retained if their $P$ values were less than $0 \cdot 05$. The missing indicator method was used to account for missing predictor data ${ }^{(25)}$.

Potential predictors were also examined as continuous variables. We explored potential non-linearity of the relationships between covariates and outcomes non-parametrically, using stepwise restricted cubic splines ${ }^{(26,27)}$. We used tests for non-linearity, using the likelihood ratio test, comparing the model with only the linear term to the model with the linear and the cubic spline terms. If non-linear associations are not reported, they were not significant.

Multivitamin supplementation was shown to significantly improve haematological outcomes in a previous analysis ${ }^{(28)}$; therefore, micronutrient regimen (multivitamins, vitamin A, multivitamins and vitamin A, or placebo) assignment was included as a covariate in all multivariate analyses.

\section{Additional analyses}

We allowed covariates to vary with time in the analyses for each of the outcomes, including measurements at each assessment during the follow-up period. We considered both the 'discrete' ${ }^{,(22)}$ and 'Breslow' ${ }^{\text {(29) }}$ options for 
ties in analyses, since the Breslow method may give biased results when the number of ties is large and risk sets are small. All results reported use the Breslow method, since no differences were observed when the discrete method was used. We calculated population-attributable fractions for risk factors for each of the outcomes, in order to estimate the number of cases of outcomes that could be averted if all of the risk factors were prevented.

\section{Pregnancy and postpartum periods}

We also conducted the aforementioned analyses separately within the pregnancy and postpartum periods, to explore predictors of haematological outcomes. For analyses in the pregnancy period, binomial regression ${ }^{(30,31)}$ was used to obtain risk ratio estimates for the predictors of each haematological endpoint at delivery. We also investigated predictors of resolution of haematological endpoints during pregnancy by running binomial regression analyses among individuals with the outcome of interest at baseline, to examine if these endpoints resolved at delivery. For analyses in the postpartum period, Cox proportional hazards models were used to examine predictors of incident haematological outcomes from delivery through the end of follow-up for all endpoints.

Statistical analyses were performed using the SAS statistical software package version $9 \cdot 2$ (SAS Institute Inc., Cary, NC, USA).

\section{Results}

The baseline characteristics of the 904 women included in the present analyses (Fig. 1) are presented in Table 1 . At the baseline assessment, $83 \%$ of women were anaemic $(\mathrm{Hb}<110 \mathrm{~g} / \mathrm{l})$ and $26 \%$ were severely anaemic $(\mathrm{Hb}<85 \mathrm{~g} / \mathrm{l})$, with a mean $\mathrm{Hb}$ level of $94 \mathrm{~g} / 1 ; 45 \%$

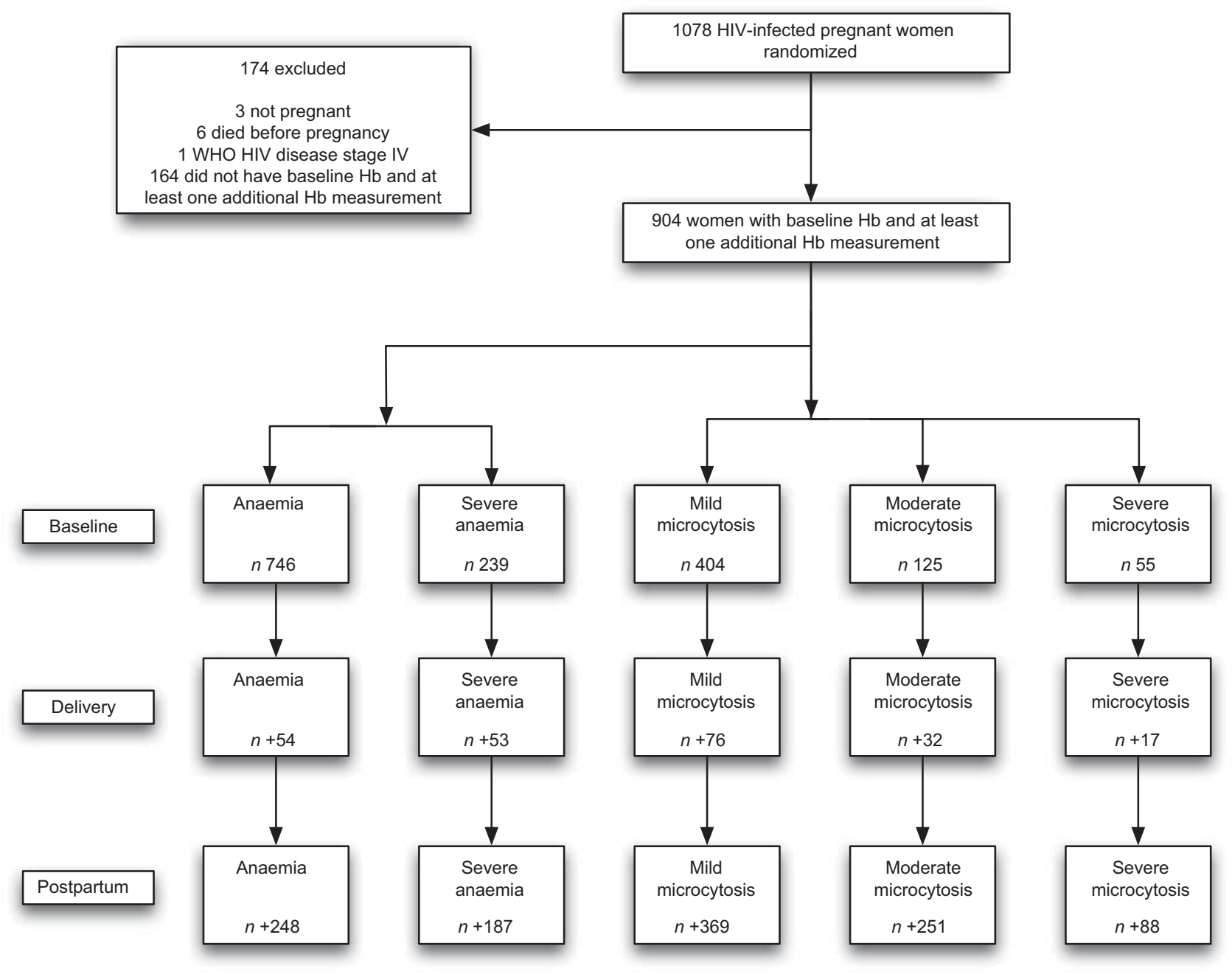

Fig. 1 Study profile of trial participants $(n$ 1078) and women $(n$ 904) included in the present analyses with available baseline and follow-up haematological measurements: HIV-infected pregnant women enrolled in a randomized trial of vitamins (1995-1997), Dar es Salaam, Tanzania. At baseline, $n$ is the number of new cases; at delivery, $n$ is the number of new cases among those who did not have the outcome at baseline; at postpartum, $n$ is the number of new cases during follow-up among those who did not have the outcome at delivery 
Table 1 Characteristics of study population: HIV-infected pregnant women ( $n$ 904) enrolled in a randomized trial of vitamins (1995-1997), Dar es Salaam, Tanzania

\begin{tabular}{|c|c|c|}
\hline Variable & Mean or $n$ & SD or \% \\
\hline \multicolumn{3}{|l|}{ Sociodemographic } \\
\hline Age (years) & $24 \cdot 7$ & $4 \cdot 8$ \\
\hline$<20$ & 117 & $12 \cdot 9$ \\
\hline $20-24$ & 368 & $40 \cdot 7$ \\
\hline $25-29$ & 271 & $30 \cdot 0$ \\
\hline$\geq 30$ & 148 & $16 \cdot 4$ \\
\hline Tanzanian shillings spent daily on food* & $1585 \cdot 7$ & $719 \cdot 9$ \\
\hline Gestational age at baseline (weeks) & $20 \cdot 4$ & $3 \cdot 4$ \\
\hline \multicolumn{3}{|l|}{ HIV-related } \\
\hline \multicolumn{3}{|l|}{ WHO HIV disease stage } \\
\hline I & 767 & $84 \cdot 8$ \\
\hline II & 128 & $14 \cdot 2$ \\
\hline III & 9 & $1 \cdot 0$ \\
\hline CD4 T-cell count (cells $/ \mu l)$ & $423 \cdot 4$ & $200 \cdot 2$ \\
\hline$<200$ & 102 & 11.9 \\
\hline 200-499 & 487 & $56 \cdot 9$ \\
\hline$\geq 500$ & 267 & $31 \cdot 2$ \\
\hline \multicolumn{3}{|l|}{ Infections } \\
\hline $\mathrm{ESR}(\mathrm{mm} / \mathrm{h})$ & $58 \cdot 3$ & $35 \cdot 6$ \\
\hline$<81$ & 620 & $74 \cdot 8$ \\
\hline$\geq 81$ & 209 & $25 \cdot 2$ \\
\hline \multicolumn{3}{|l|}{ Malaria infection } \\
\hline No & 732 & 81.9 \\
\hline Yes & 162 & $18 \cdot 1$ \\
\hline \multicolumn{3}{|l|}{ Pathogenic protozoan infectionst } \\
\hline No & 687 & $93 \cdot 2$ \\
\hline Yes & 50 & $6 \cdot 8$ \\
\hline \multicolumn{3}{|l|}{ Hookworm } \\
\hline No & 650 & $88 \cdot 4$ \\
\hline Yes & 85 & $11 \cdot 6$ \\
\hline \multicolumn{3}{|l|}{ Any helminth infectionł } \\
\hline No & 599 & $81 \cdot 3$ \\
\hline Yes & 138 & $18 \cdot 7$ \\
\hline \multicolumn{3}{|l|}{$\begin{array}{l}\text { Any intestinal parasite (pathogenic } \\
\text { protozoan or helminth infection) }\end{array}$} \\
\hline No & 557 & $75 \cdot 6$ \\
\hline Yes & 180 & $24 \cdot 4$ \\
\hline \multicolumn{3}{|l|}{ Syphilis } \\
\hline No & 721 & $94 \cdot 0$ \\
\hline Yes & 46 & $6 \cdot 0$ \\
\hline \multicolumn{3}{|l|}{ Vaginal candidiasis } \\
\hline No & 527 & $59 \cdot 8$ \\
\hline Yes & 355 & $40 \cdot 2$ \\
\hline \multicolumn{3}{|l|}{ Sexually transmitted infections } \\
\hline No & 651 & $72 \cdot 4$ \\
\hline Yes & 248 & $27 \cdot 6$ \\
\hline \multicolumn{3}{|l|}{ Nutritional } \\
\hline BMI $\left(\mathrm{kg} / \mathrm{m}^{2}\right)$ & $23 \cdot 4$ & $3 \cdot 3$ \\
\hline Plasma vitamin $\mathrm{D}$ concentration $(\mathrm{ng} / \mathrm{ml})$ & $36 \cdot 5$ & $12 \cdot 1$ \\
\hline$<32$ & 275 & $37 \cdot 4$ \\
\hline $\mathrm{Hb}$ concentration $(\mathrm{g} / \mathrm{l})$ & 94 & $17 \cdot 0$ \\
\hline$<110$ & 746 & $82 \cdot 5$ \\
\hline$<85$ & 239 & $26 \cdot 4$ \\
\hline
\end{tabular}

ESR, erythrocyte sedimentation rate.

Data are presented as mean and SD for continuous variables or as $n$ and \% for categorical variables.

*\$US 1 was equivalent to approximately 500 Tanzanian Shillings at the time of data collection.

+Giardia lamblia, Entamoeba histolytica or Cryptosporidium parvum. ¥Hookworm, Trichuris trichura, Ascaris lumbricoides, Strongyloides stercoralis or Schistosoma mansoni.

and $14 \%$ of women had mild or moderate hypochromic microcytosis, respectively. In contrast, only $6 \%$ of women had severe microcytosis. Tables 2 to 5 present results only for anaemia, severe anaemia and severe microcytosis, except for resolution of anaemia; for the resolution of anaemia analyses, results are presented for mild microcytosis, as all cases of severe microcytosis resolved by delivery.

A total of $87 \%$ of women developed incident anaemia over the follow-up period. Infection with malaria and pathogenic protozoa were associated with significant twofold increases in the risk of developing anaemia during follow-up (Table 2). In contrast, higher Hb concentrations were associated with lower risk of anaemia during follow-up, with a significant $25 \%$ lower risk per 10 unit (g/l) increase in baseline $\mathrm{Hb}$ concentrations. A total of 222 women out of 667 at risk developed severe anaemia during follow-up. Women with higher ESR ( $\geq 81 \mathrm{~mm} / \mathrm{h}$ ) and higher CD8 T-cell counts had significantly increased risk of developing severe anaemia; low vitamin D status $(<32 \mathrm{ng} / \mathrm{ml})$ was associated with a $41 \%$ significant increase in the risk of severe anaemia during follow-up. In contrast, higher CD4 T-cell counts predicted lower risk of severe anaemia, with a 13\% lower risk per 100-cell increase in baseline CD4 T-cells. A significant non-linear relationship was observed between baseline CD4 T-cell counts and $\mathrm{Hb}$ concentrations $(P<0 \cdot 01) . \mathrm{Hb}$ concentrations increased with $\mathrm{CD} 4 \mathrm{~T}$-cell counts until approximately CD 4 count of $700 \mathrm{cells} / \mu \mathrm{l}$, and then decreased after this point. A second pregnancy after enrolment in the trial was associated with $51 \%$ reduction in the risk of anaemia during the follow-up period.

A total of $69 \%, 38 \%$ and $15 \%$ women developed incident mild, moderate and severe hypochromic microcytosis during follow-up, respectively. The majority of microcytosis cases developed during the postpartum period. Increased CD8 T-cell, lower CD4 T-cell and lower $\mathrm{Hb}$ concentrations at baseline significantly predicted elevated risk of severe hypochromic microcytosis. Hookworm and syphilis infections were associated with a significant 1.9 - and $2 \cdot 3$-fold increase in the risk of severe microcytosis during follow-up. Low vitamin $\mathrm{D}$ status was associated with a significant $2 \cdot 4$-fold increase in the risk of severe microcytosis.

A total of 125 women who were not anaemic at baseline also had $\mathrm{Hb}$ measures available at delivery; out of these, fifty-four became anaemic during pregnancy. In the pregnancy period, higher $\mathrm{Hb}$ concentrations were associated with significantly reduced risk of incident anaemia and severe anaemia at delivery (Table 3). Women with low vitamin D status at baseline also had a higher risk of developing severe anaemia or hypochromic microcytosis at delivery. Further, candidiasis was associated with increased risk of severe hypochromic microcytosis.

In analyses of population-attributable fractions, we estimated the number of cases of outcomes that could be averted if the selected risk factor was prevented. For anaemia, the population-attributable fraction for malaria was $8.6 \%$; approximately $9 \%$ of anaemia cases would be averted if all of the malaria cases were prevented. For severe hypochromic microcytosis, the population-attributable fraction for hookworm was $6 \cdot 2 \%$; approximately $6 \%$ of 
Table 2 Predictors ${ }^{\star}$ of haematological outcomes during the overall follow-up period (including the delivery and postpartum periods): HIV-infected pregnant women enrolled in a randomized trial of vitamins (1995-1997), Dar es Salaam, Tanzania

\begin{tabular}{|c|c|c|c|c|c|c|c|c|c|}
\hline \multirow[b]{2}{*}{ Variable } & \multicolumn{3}{|c|}{ Anaemiat ( $n / N$ 138/158) } & \multicolumn{3}{|c|}{ Severe anaemiat ( $n / N 222 / 667)$} & \multicolumn{3}{|c|}{ Severe microcytosist ( $n / N$ 125/850 } \\
\hline & $\mathrm{HR}$ & $95 \% \mathrm{Cl}$ & $P$ value & $\mathrm{HR}$ & $95 \% \mathrm{Cl}$ & $P$ valueł & $\mathrm{HR}$ & $95 \% \mathrm{Cl}$ & $P$ value $\ddagger$ \\
\hline \multicolumn{10}{|l|}{ Immunological } \\
\hline CD4 T-cell count (per 100 cells) & - & - & - & 0.87 & $0.81,0.94$ & $<0.001$ & 0.82 & $0.73,0.91$ & $<0.001$ \\
\hline CD8 T-cell count (per 100 cells) & - & - & - & 1.07 & $1 \cdot 02,1 \cdot 11$ & $<0.01$ & $1 \cdot 08$ & $1 \cdot 02,1 \cdot 14$ & $<0.01$ \\
\hline \multicolumn{10}{|l|}{ Nutritional } \\
\hline $\mathrm{Hb}($ per $10 \mathrm{~g} / \mathrm{l})$ & 0.75 & $0.58,0.96$ & 0.02 & - & - & - & $0 \cdot 77$ & $0.67,0.87$ & $<0.0001$ \\
\hline Low vitamin $\mathrm{D}(<32 \mathrm{ng} / \mathrm{ml})$ & - & - & - & $1 \cdot 41$ & $1 \cdot 05,1 \cdot 89$ & 0.02 & $2 \cdot 38$ & $1 \cdot 58,3 \cdot 58$ & $<0.0001$ \\
\hline \multicolumn{10}{|l|}{ Infections } \\
\hline High ESR $(\geq 81 \mathrm{~mm} / \mathrm{h})$ & - & - & - & 1.53 & $1 \cdot 112,2 \cdot 08$ & $<0.01$ & - & - & - \\
\hline Hookworm & - & - & - & - & - & - & 1.94 & $1 \cdot 13,3 \cdot 35$ & 0.02 \\
\hline Malaria & 1.97 & $1 \cdot 23,3 \cdot 15$ & $<0.01$ & _ & _ & - & - & - & - \\
\hline Pathogenic protozoa§ & $1 \cdot 83$ & $1 \cdot 00,3 \cdot 37$ & 0.05 & - & - & - & - & - & - \\
\hline Syphilis & - & - & - & - & - & - & $2 \cdot 34$ & $1 \cdot 15,4 \cdot 74$ & 0.02 \\
\hline \multicolumn{10}{|l|}{ Additional variables } \\
\hline Second pregnancy\| & 0.49 & $0.29,0.83$ & $<0.01$ & $0 \cdot 41$ & $0.30,0.58$ & $<0.0001$ & $0 \cdot 47$ & $0 \cdot 31,0 \cdot 70$ & $<0.001$ \\
\hline
\end{tabular}

HR, hazard ratio; ESR, erythrocyte sedimentation rate.

${ }^{*}$ All multivariate analyses were adjusted for multivitamin regimen and adherence to prenatal Fe supplementation.

†Anaemia: $\mathrm{Hb}<110 \mathrm{~g} / \mathrm{l}$, severe anaemia: $\mathrm{Hb}<85 \mathrm{~g} / \mathrm{l}$, severe microcytosis: hypochromasia $\geq 2+$ and microcytic cells on peripheral smear.

$\ddagger P$ values were obtained from Cox proportional hazards analyses; women who had the endpoint of interest at baseline were excluded, to examine time to first occurrence of outcome during follow-up.

§Giardia lamblia, Entamoeba histolytica or Cryptosporidium parvum.

॥Second pregnancy after enrolment into the trial.

Table 3 Predictors* of haematological outcomes during pregnancy (incident cases at delivery): HIV-infected pregnant women enrolled in a randomized trial of vitamins (1995-1997), Dar es Salaam, Tanzania

\begin{tabular}{|c|c|c|c|c|c|c|c|c|c|}
\hline \multirow[b]{2}{*}{ Variable } & \multicolumn{3}{|c|}{ Anaemiat ( $n / N 54 / 125)$} & \multicolumn{3}{|c|}{ Severe anaemiat ( $n / N 53 / 534)$} & \multicolumn{3}{|c|}{ Severe microcytosist $(n / N$ 17/673) } \\
\hline & RR & $95 \% \mathrm{Cl}$ & $P$ valueł & $\mathrm{RR}$ & $95 \% \mathrm{Cl}$ & $P$ value $\ddagger$ & $\mathrm{RR}$ & $95 \% \mathrm{Cl}$ & $P$ value \\
\hline \multicolumn{10}{|l|}{ Nutritional } \\
\hline $\mathrm{Hb}($ per $10 \mathrm{~g} / \mathrm{l})$ & $0 \cdot 70$ & $0.51,0.96$ & 0.03 & $0 \cdot 75$ & $0.58,0.98$ & 0.03 & - & - & - \\
\hline Low vitamin $\mathrm{D}(<32 \mathrm{ng} / \mathrm{ml})$ & - & - & - & $1 \cdot 84$ & $1 \cdot 04,3 \cdot 28$ & 0.04 & $3 \cdot 20$ & $1 \cdot 04,9 \cdot 85$ & 0.04 \\
\hline Infections & & & & & & & & & \\
\hline Candidiasis & - & - & - & - & - & - & $3 \cdot 16$ & $1 \cdot 07,9 \cdot 36$ & 0.04 \\
\hline
\end{tabular}

$\mathrm{RR}$, relative risk.

*All multivariate analyses were adjusted for multivitamin regimen and adherence to prenatal Fe supplementation.

tAnaemia: $\mathrm{Hb}<110 \mathrm{~g} / \mathrm{l}$, severe anaemia: $\mathrm{Hb}<85 \mathrm{~g} / \mathrm{l}$, severe microcytosis: hypochromasia $\geq 2+$ and microcytic cells on peripheral smear.

$\ddagger P$ values were obtained from binomial regression analyses; women who had the endpoint of interest at baseline were excluded, to examine the occurrence of each outcome at delivery.

anaemia cases would be averted if all of the hookworm cases were prevented through antihelminthic treatment.

Resolution of anaemia at delivery occurred in 251 women out of the 590 women who had anaemia at baseline (Table 4). Low vitamin D concentrations and candidiasis were associated with significantly reduced likelihood of resolution of anaemia. For severe anaemia and microcytosis, primiparity was associated with greater likelihood of resolution.

Among the 323 women who did not have anaemia at delivery, 248 became anaemic during postpartum follow-up (Table 5). Higher baseline $\mathrm{Hb}$ concentrations, increased money spent on food and higher CD4 T-cell counts were associated with a significant independent lower risk of anaemia during follow-up, whereas infection with malaria was associated with a significant 1.6 times greater risk of anaemia during the postpartum period. No formal education, higher CD8 T-cell counts and candidiasis were associated with significantly increased risk of severe anaemia during follow-up, whereas higher baseline $\mathrm{Hb}$ concentrations and CD4 T-cell counts were associated with significantly lower risk of severe anaemia during the postpartum period. Low vitamin D status was associated with a significant two-fold increase in the risk of severe microcytosis; higher baseline $\mathrm{Hb}$ concentrations and CD4 T-cell counts were associated with significantly reduced risk of severe microcytosis during the postpartum period. A second pregnancy after enrolment in the trial was also associated with decreased risk of severe microcytosis during the postpartum period.

\section{Discussion}

In the present study, parasitic infections, low CD4 T-cell counts, high ESR and vitamin D insufficiency were the 
Table 4 Predictors ${ }^{*}$ of resolution of haematological outcomes during pregnancy (resolution of cases at delivery): HIV-infected pregnant women enrolled in a randomized trial of vitamins (1995-1997), Dar es Salaam, Tanzania

\begin{tabular}{|c|c|c|c|c|c|c|c|c|c|}
\hline \multirow[b]{2}{*}{ Variable } & \multicolumn{3}{|c|}{ Anaemiat (n/N 251/590) } & \multicolumn{3}{|c|}{ Severe anaemiat (n/N 157/181) } & \multicolumn{3}{|c|}{ Mild microcytosist ( $n / N$ 230/315) } \\
\hline & $\mathrm{RR}$ & $95 \% \mathrm{Cl}$ & $P$ value $\ddagger$ & $\mathrm{RR}$ & $95 \% \mathrm{Cl}$ & $P$ valueł & $\mathrm{RR}$ & $95 \% \mathrm{Cl}$ & $P$ value $\neq$ \\
\hline \multicolumn{10}{|l|}{ Nutritional } \\
\hline $\begin{array}{l}\text { Low vitamin D (<32 ng/ml) } \\
\text { Infection }\end{array}$ & $0 \cdot 78$ & $0.63,0.98$ & 0.03 & - & - & - & - & - & - \\
\hline $\begin{array}{l}\text { Candidiasis } \\
\text { Additional variables }\end{array}$ & $0 \cdot 78$ & $0.64,0.96$ & 0.01 & - & - & - & - & - & - \\
\hline Primiparous & - & - & - & $1 \cdot 16$ & $1 \cdot 04,1 \cdot 30$ & $<0.01$ & $1 \cdot 18$ & $1 \cdot 04,1 \cdot 34$ & $<0.01$ \\
\hline
\end{tabular}

RR, relative risk.

Note: all baseline severe microcytosis cases and all but five moderate microcytosis cases resolved at delivery.

*All multivariate analyses were adjusted for multivitamin regimen and adherence to prenatal Fe supplementation.

†Anaemia: $\mathrm{Hb}<110 \mathrm{~g} / \mathrm{l}$, severe anaemia: $\mathrm{Hb}<85 \mathrm{~g} / \mathrm{l}$, mild microcytosis: hypochromasia $\geq 1+$ with or without microcytosis on peripheral smear.

$\ddagger P$ values were obtained from binomial regression analyses; analyses were conducted among women with the outcome of interest at baseline, to examine resolution of outcome at delivery.

Table 5 Predictors* of postpartum haematological outcomes: HIV-infected pregnant women enrolled in a randomized trial of vitamins (1995-1997), Dar es Salaam, Tanzania

\begin{tabular}{|c|c|c|c|c|c|c|c|c|c|}
\hline \multirow[b]{2}{*}{ Variable } & \multicolumn{3}{|c|}{$\begin{array}{c}\text { Anaemiat } \\
(n / N 248 / 323)\end{array}$} & \multicolumn{3}{|c|}{$\begin{array}{l}\text { Severe anaemiat } \\
(n / N 187 / 640)\end{array}$} & \multicolumn{3}{|c|}{$\begin{array}{l}\text { Severe microcytosist } \\
(n / N 88 / 700)\end{array}$} \\
\hline & HR & $95 \% \mathrm{Cl}$ & $P$ value $\ddagger$ & $\mathrm{HR}$ & $95 \% \mathrm{Cl}$ & $P$ value $\ddagger$ & $\mathrm{HR}$ & $95 \% \mathrm{Cl}$ & $P$ value \\
\hline \multicolumn{10}{|l|}{ Sociodemographic } \\
\hline No formal education & - & - & - & $2 \cdot 26$ & $1 \cdot 37,3 \cdot 70$ & $<0.01$ & - & - & - \\
\hline Tanzanian shillings spent on food (per 1000/d) & 0.74 & $0.60,0.91$ & $<0.01$ & - & - & - & - & - & - \\
\hline \multicolumn{10}{|c|}{ Immunological } \\
\hline CD4 T-cell count (per 100 cells) & 0.93 & $0.87,0.99$ & $0 \cdot 02$ & $0 \cdot 85$ & $0 \cdot 78,0.93$ & $<0.001$ & $0 \cdot 87$ & $0 \cdot 76,0.98$ & 0.03 \\
\hline CD8 T-cell count (per 100 cells) & - & - & - & 1.09 & $1 \cdot 04,1 \cdot 14$ & $<0.001$ & - & - & - \\
\hline \multicolumn{10}{|l|}{ Nutritional } \\
\hline $\mathrm{Hb}($ per $10 \mathrm{~g} / \mathrm{l})$ & $0 \cdot 87$ & $0.81,0.94$ & $<0.001$ & $0 \cdot 81$ & $0 \cdot 74,0 \cdot 88$ & $<0.0001$ & $0 \cdot 76$ & $0 \cdot 67,0.86$ & $<0.0001$ \\
\hline Low vitamin D (<32 ng/ml) & - & - & - & - & - & - & 1.97 & $1 \cdot 21,3 \cdot 20$ & $<0.01$ \\
\hline \multicolumn{10}{|l|}{ Infections } \\
\hline Malaria & $1 \cdot 59$ & $1 \cdot 14,2 \cdot 23$ & $<0.01$ & - & - & - & - & - & - \\
\hline Candidiasis & - & - & - & $1 \cdot 41$ & $1 \cdot 05,1 \cdot 89$ & 0.02 & - & - & - \\
\hline \multicolumn{10}{|l|}{ Additional variables } \\
\hline Second pregnancy§ & 0.47 & $0.34,0.65$ & $<0.0001$ & 0.54 & $0 \cdot 39,0 \cdot 74$ & $<0.001$ & 0.57 & $0.36,0.90$ & 0.02 \\
\hline
\end{tabular}

HR, hazard ratio.

*All multivariate analyses were adjusted for multivitamin regimen and adherence to prenatal Fe supplementation.

†Anaemia: $\mathrm{Hb}<110 \mathrm{~g} / \mathrm{l}$, severe anaemia: $\mathrm{Hb}<85 \mathrm{~g} / \mathrm{l}$, severe microcytosis: hypochromasia $\geq 2+$ and microcytic cells on peripheral smear.

$\ddagger P$ values were obtained from Cox proportional hazards analyses; women who had the endpoint of interest at baseline (delivery) were excluded, to examine time to the first occurrence of each outcome during follow-up.

$\S$ Second pregnancy after enrolment into the trial.

main predictors of anaemia and Fe deficiency in HIVinfected pregnant women in Tanzania.

Our analysis is distinct from previous studies due to its longitudinal prospective evaluation of incident haematological outcomes, extensive assessment of potential risk factors, and investigation of predictors of resolution of haematological outcomes in a cohort of HIV-infected pregnant women receiving Fe-folate supplementation. Women were followed for a long duration through the postpartum period, with a median of 57 months, with an average of $8.6 \mathrm{Hb}$ measurements per participant during follow-up.

The prevalence of anaemia and hypochromic microcytosis in this population was high and similar to other studies in HIV-infected pregnant women in Sub-Saharan Africa $^{(3-5,32,33)}$. For example, several studies have reported an extremely high prevalence of anaemia $(\mathrm{Hb}<110 \mathrm{~g} / \mathrm{l})$ in
HIV-infected pregnant women: $78 \%$ in Burkina $\mathrm{Faso}^{(3)}$, $83 \%$ in Côte d'Ivoire ${ }^{(4)}$ and $73 \%$ in an analysis of a multicentre trial in Tanzania, Zambia and Malawi ${ }^{(5)}$.

Similar to our findings, malaria ${ }^{(34)}$, hookworm ${ }^{(35)}$ and other infections, such as schistosomiasis and trichuriasis ${ }^{(6)}$, have been identified as important contributors to the high prevalence of anaemia and Fe deficiency in many resourcelimited settings. In the current analysis, malaria infection at baseline predicted a two-fold increase in the risk of anaemia during follow-up and a 1.6 times greater risk of anaemia during the postpartum period. In a previous cross-sectional analysis, women with high malaria parasite density $(\geq 1000$ parasites $/ \mu$ l) had $2 \cdot 7$-fold greater odds of severe anaemia (95\% CI $1 \cdot 6,4 \cdot 6 ; P=0.0003$ ) at baseline, compared with uninfected women ${ }^{(2)}$.

The relationship between parasitic worm infections and anaemia has not been evaluated in HIV-infected 
pregnant women. However, the relationship between parasitic worm infections, particularly hookworm infection, and anaemia has been well established in children ${ }^{(36)}$. Further, in a meta-analysis by Brooker et al. of thirteen studies among presumably HIV-uninfected pregnant women, hookworm infection was significantly associated with anaemia and infection intensity was significantly inversely related to $\mathrm{Hb}$ concentrations ${ }^{(37)}$. These infections, along with malaria, may contribute to Fe deficiency via destruction of erythrocytes, occult blood loss and nutrient malabsorption ${ }^{(36)}$.

Higher CD8 T-cell counts and ESR, and lower CD4 T-cell concentrations, predicted increased risk of anaemia and Fe deficiency in the present study, possibly due to associated increase in inflammation. Although the conventional role of CD8 T-cells is as cytotoxic killer cells, they may also be effector cells in inflammation ${ }^{(38-42)}$ and lead to anaemia. HIV infection and advanced HIV disease may itself contribute to the aetiology of anaemia and Fe deficiency through a number of mechanisms, such as infection of marrow stromal cells ${ }^{(43)}$, impaired haematopoietic progenitor cell growth ${ }^{(44)}$, bone marrow pathologies, autoimmune haemolysis and intestinal blood loss ${ }^{(45-47)}$. Anaemia of inflammation ${ }^{(48)}$ is a leading cause of anaemia in HIV infection ${ }^{(13,48)}$ and is characterized by a distinctive haematological profile and a lack of response to $\mathrm{Fe}$ supplementation $^{(49)}$.

The relationship between vitamin D and Fe status has not previously been evaluated in the context of pregnancy or HIV infection. However, findings are consistent with a previous analysis in the TOV in which low baseline vitamin D status was associated with decreased risk of anaemia during overall follow-up ${ }^{(50)}$. In the present analysis we demonstrated that low baseline vitamin D concentrations predicted risk of anaemia during the pregnancy and postpartum periods, and adequate vitamin D status was an important predictor of resolution of anaemia and Fe deficiency. There are several plausible biological mechanisms by which vitamin D could modulate risk of anaemia, such as through decreasing inflammation. Vitamin D deficiency has also been associated with marrow myelofibrosis, which is a known cause of anaemia ${ }^{(51)}$. An association between low vitamin D status and Fe deficiency has been observed in earlier studies in individuals with renal disease in the third National Health and Nutrition Examination Survey ${ }^{(52)}$ and among children in Bangladeshi, Pakistani and Indian ${ }^{(53)}$, and Asian ${ }^{(54)}$ communities in Britain. However, there is a lack of evidence regarding aetiological mechanisms and the relationship between vitamin D and Fe status needs to be further explored in pregnant women and in resourcelimited settings.

Risk of microcytosis was inversely associated with the daily amount of household funds allocated to food and educational level. This is consistent with previous studies that identified poverty as a risk factor for Fe deficiency and the increased prevalence of anaemia in resource-limited settings. This may be related to the relatively higher cost of Fe-rich food sources, such as animal products, nuts and green leafy vegetables, compared with grain-based staples. In developing settings, bioavailable haem Fe may comprise as little as $5 \%$ of the diet, compared with 18 to $25 \%$ in adults consuming typically Western diets ${ }^{(55)}$.

Of note is the high number of baseline cases of anaemia $(42 \cdot 5 \%)$, severe anaemia $(86 \cdot 7 \%)$ and microcytosis $(73.0 \%)$ that resolved at delivery, with relatively high numbers of new cases of anaemia $(76 \cdot 8 \%)$, severe anaemia (29.2\%) and mild (66.5\%), moderate (36.9\%) and severe (12.6\%) Fe deficiency developing during the postpartum period, after discontinuation of prenatal Fe supplementation. These results suggest that Fe supplementation only during pregnancy may not be sufficient to prevent anaemia and Fe deficiency during the postpartum period.

Interestingly, a second pregnancy after enrolment was associated with marked reductions in risk of haematological outcomes during overall follow-up and the postpartum period. This finding may be attributable to reverse causation, i.e. women with better haematological or nutritional status were more likely to become pregnant a second time during the follow-up period. Although a second pregnancy may be associated with adverse pregnancy outcomes in resource-limited settings due to depletion of maternal reserves, it also presents an opportunity for greater contact with the health-care system. In previous publications, we have noted a significantly lower incidence of anaemia in women who received multivitamin (vitamins B, C, E) supplementation. The combination of multivitamins received during the trial, plus Fe and folate supplementation during pregnancy and access to health care, may synergistically reduce the risks of adverse haematological outcomes in this setting.

Our study has a few limitations. The use of $\mathrm{Hb}$ and hypochromic microcytosis as the only indicators of $\mathrm{Fe}$ status is a limitation. Although $\mathrm{Hb}$ is the most common indicator for anaemia worldwide, it does not reflect $\mathrm{Fe}$ stores and has low sensitivity. Similarly, hypochromic microcytosis does not reflect available body Fe stores. Physiological changes in pregnancy, particularly haemodilution, also affect the concentrations and interpretation of $\mathrm{Fe}$ indicators. The lack of recording of alternative indicators of Fe status (such as serum ferritin and soluble transferrin receptor) and inflammation (such as C-reactive protein or $\alpha-1$ acid glycoprotein) are limitations in our analysis. According to WHO, where possible, concentrations of $\mathrm{Hb}$, serum ferritin and transferrin receptor, and at least one acute-phase protein should be measured ${ }^{(56)}$. For example, acute-phase reactants such as C-reactive protein and $\alpha-1$ acid glycoprotein would further improve $\mathrm{Fe}$ assessment in the context of inflammation and infection; recent research has also identified hepcidin as an important regulator of $\mathrm{Fe}$ metabolism in anaemia of inflammation.

The present analysis was also conducted to examine predictors of anaemia among HIV-infected women who 
were not on ART; as such, findings may not be generalizable to pregnant women receiving ART. Scale-up of ART and prenatal services in many developing settings may have reduced the prevalence of anaemia in HIV-infected populations. However, using baseline characteristics may inform prevention and clinical management of anaemia during pregnancy and the follow-up period among women who have not yet been initiated on ART. Further research is also needed to examine novel biomarkers, such as hepcidin, and to explore the relationships of these predictors with anaemia in HIV-infected individuals on ART.

Fe supplementation alone, and particularly only during pregnancy, may not be adequate to prevent anaemia and associated morbidities in HIV-infected individuals. Consideration of other aetiological factors, namely HIV, malaria, hookworm and other endemic infectious diseases, and underlying nutritional deficiencies; infectious disease control; and micronutrient supplementation, are needed in the prevention, screening and clinical management of anaemia and Fe deficiency in HIV-infected women during the pre- and postpartum periods. Further research is also needed to examine the interactions of these risk factors among individuals on ART.

\section{Acknowledgements}

The study was supported by the National Institute of Child Health and Human Development (NICHD R01 32257; and K24HD058795) and the Harvard School of Public Health. None of the authors had a personal or financial conflict of interest. J.L.F., S.M., C.P.D., D.S. and W.W.F. contributed to the plans for data analysis. J.L.F. analysed and interpreted the data and wrote the initial draft of the manuscript. S.M. assisted with the interpretation of data. D.S. provided statistical guidance and helped interpret data analyses. G.I.M., S.A., D.S. and W.W.F. were investigators of the trial and contributed to the study design and implementation. All co-authors participated in manuscript preparation. The authors are grateful to the mothers and children, also the field teams, including physicians, nurses, midwives, supervisors, laboratory staff and the administrative staff, who made this study possible; and Muhimbili Medical Centre, Muhimbili University College of Health Sciences, and the National AIDS Control Program in Dar es Salaam for their institutional support.

\section{References}

1. World Health Organization (2008) Worldwide Prevalence of Anaemia 1993-2005. WHO Global Database on Anaemia. Geneva: WHO.

2. Antelman G, Msamanga GI, Spiegelman D et al. (2000) Nutritional factors and infectious disease contribute to anemia among pregnant women with human immunodeficiency virus in Tanzania. J Nutr 130, 1950-1957.
3. Meda N, Dao B \& Ouangre A (1998) HIV, maternal anemia and perinatal intervention using zidovudine. DITRAME Study Group (ANRS 049 Clinical Trial). Int J Gynaecol Obstet 61, 65-66.

4. Ramon R, Sawadogo D, Koko FS et al. (1999) Haematological characteristics and HIV status of pregnant women in Abidjan, Cote d'Ivoire, 1995-1996. Trans R Soc Trop Med Hyg 93, 419-422.

5. Mehta S, Manji KP, Young AM et al. (2008) Nutritional indicators of adverse pregnancy outcomes and motherto-child transmission of HIV among HIV-infected women. Am J Clin Nutr 87, 1639-1649.

6. World Health Organization/UNICEF/United Nations University (2001) Iron Deficiency Anaemia: Assessment, Prevention, and Control. Geneva: WHO.

7. Stoltzfus RJ (2001) Iron-deficiency anemia: reexamining the nature and magnitude of the public health problem. Summary: implications for research and programs. J Nutr 131, 2 Suppl. 2, 697S-700S.

8. Allen LH (2000) Anemia and iron deficiency: effects on pregnancy outcome. Am J Clin Nutr 71, 5 Suppl., 1280S-1284S.

9. Brabin BJ, Hakimi M \& Pelletier D (2001) An analysis of anemia and pregnancy-related maternal mortality. $J$ Nutr 131, 2 Suppl. 2, 604S-614S

10. Stivelman JC (2000) Benefits of anaemia treatment on cognitive function. Nephrol Dial Transplant 15, Suppl. 3, $29-35$.

11. Haas JD \& Brownlie T (2001) Iron deficiency and reduced work capacity: a critical review of the research to determine a causal relationship. J Nutr 131, 2 Suppl. 2, 676S-688S.

12. Mungen E (2003) Iron supplementation in pregnancy. $J$ Perinat Med 31, 420-426.

13. Weiss G \& Goodnough LT (2005) Anemia of chronic disease. N Engl J Med 352, 1011-1023.

14. World Health Organization/UNICEF (2004) Focusing on Anaemia. Towards an Integrated Approach for Effective Anaemia Control. Geneva: WHO.

15. Fawzi WW, Msamanga GI, Spiegelman D et al. (1999) Rationale and design of the Tanzania Vitamin and HIV Infection Trial. Control Clin Trials 20, 75-90.

16. The WHO International Collaborating Group for the Study of the WHO Staging System (1993) Proposed 'World Health Organization staging system for HIV infection and disease': preliminary testing by an international collaborative crosssectional study. AIDS 7, 711-718.

17. Urassa W, Matunda S, Bredberg-Raden U et al. (1994) Evaluation of the WHO human immunodeficiency virus (HIV) antibody testing strategy for the diagnosis of HIV infection. Clin Diagn Virol 2, 1-6.

18. World Health Organization (1991) Basic Laboratory Methods in Medical Parasitology. Geneva: WHO.

19. Hollis BW (2005) Circulating 25-hydroxyvitamin D levels indicative of vitamin D sufficiency: implications for establishing a new effective dietary intake recommendation for vitamin D. J Nutr 135, 317-322.

20. Mehta S, Hunter DJ, Mugusi FM et al. (2009) Perinatal outcomes, including mother-to-child transmission of HIV, and child mortality and their association with maternal vitamin D status in Tanzania. J Infect Dis 200, 1022-1030.

21. Dacie JV \& Lewis SM (1991) Practical Hematology. Harlow: Longman Group UK Ltd.

22. Cox D (1972) Regression models and life tables. J R Stat Soc 34, 187-220.

23. Antelman G, Smith-Fawzi M, Kaaya S et al. (2001) Predictors of HIV-1 sero-status disclosure: a prospective study among HIV infected pregnanat women in Dar es Salaam, Tanzania. AIDS 15, 1865-1874.

24. World Health Organization (1995) Physical Status: The Use and Interpretation of Anthropometry. Report of a WHO Expert Committee. Geneva: WHO. 
25. Miettinen O (1985) Theoretical Epidemiology. New York: John Wiley \& Sons.

26. Durrleman S \& Simon R (1989) Flexible regression models with cubic splines. Stat Med $\mathbf{8}, 551-561$.

27. Govindarajulu US, Spiegelman D, Thurston SW et al. (2007) Comparing smoothing techniques in Cox models for exposure-response relationships. Stat Med 26, 3735-3752.

28. Fawzi WW, Msamanga GI, Kupka R et al. (2007) Multivitamin supplementation improves hematologic status in HIV-infected women and their children in Tanzania. Am J Clin Nutr 85, 1335-1343.

29. Breslow N (1974) Covariance analysis of censored survival data. Biometrics 30, 89-99.

30. Wacholder S (1986) Binomial regression in GLIM: estimating risk ratios and risk differences. Am J Epidemiol 123, 174-184.

31. Spiegelman D \& Hertzmark E (2005) Easy SAS calculations for risk or prevalence ratios and differences. $A m J$ Epidemiol 162, 199-200.

32. Friis H, Gomo E, Kastel P et al. (2001) HIV and other predictors of serum folate, serum ferritin, and hemoglobin in pregnancy: a cross-sectional study in Zimbabwe. $A m \mathrm{~J}$ Clin Nutr 73, 1066-1073.

33. Semba RD, Kumwenda N, Hoover DR et al. (2000) Assessment of iron status using plasma transferrin receptor in pregnant women with and without human immunodeficiency virus infection in Malawi. Eur J Clin Nutr 54, 872-877.

34. Fawzi WW, Msamanga G, Hunter D et al. (2000) Randomized trial of vitamin supplements in relation to vertical transmission of HIV-1 in Tanzania. J Acquir Immune Defic Syndr 23, 246-254.

35. Stoltzfus RJ, Chwaya HM, Tielsch JM et al. (1997) Epidemiology of iron deficiency anemia in Zanzibari schoolchildren: the importance of hookworms. Am J Clin Nutr 65, 153-159.

36. Brooker S, Akhwale W, Pullan R et al. (2007) Epidemiology of plasmodium-helminth co-infection in Africa: populations at risk, potential impact on anemia, and prospects for combining control. Am J Trop Med Hyg 77, 88-98.

37. Brooker S, Hotez PJ \& Bundy DA (2008) Hookwormrelated anaemia among pregnant women: a systematic review. PLoS Negl Trop Dis 2, e291.

38. Meehan TF \& DeLuca HF (2002) CD8(+) T cells are not necessary for $1 \alpha, 25$-dihydroxyvitamin $\mathrm{D}(3)$ to suppress experimental autoimmune encephalomyelitis in mice. Proc Natl Acad Sci USA 99, 5557-5560.

39. Babbe H, Roers A, Waisman A et al. (2000) Clonal expansions of CD8(+) T cells dominate the T cell infiltrate in active multiple sclerosis lesions as shown by micromanipulation and single cell polymerase chain reaction. J Exp Med 192, 393-404.

40. de Vos AF, Dick AD, Klooster J et al. (2000) Analysis of the cellular infiltrate in the iris during experimental autoimmune encephalomyelitis. Invest Ophthalmol Vis Sci 41, 3001-3010.
41. Dressel A, Chin JL, Sette A et al. (1997) Autoantigen recognition by human CD8 T cell clones: enhanced agonist response induced by altered peptide ligands. I Immunol 159, 4943-4951.

42. Monteiro J, Hingorani R, Peroglizzi R et al. (1996) Oligoclonality of $\mathrm{CD}^{+} \mathrm{T}$ cells in multiple sclerosis. Autoimmunity 23, 127-138.

43. Koka PS, Jamieson BD, Brooks DG et al. (1999) Human immunodeficiency virus type 1 -induced hematopoietic inhibition is independent of productive infection of progenitor cells in vivo. J Virol 73, 9089-9097.

44. Moses AV, Williams S, Heneveld ML et al. (1996) Human immunodeficiency virus infection of bone marrow endothelium reduces induction of stromal hematopoietic growth factors. Blood $\mathbf{8 7}$, 919-925.

45. Coyle TE (1997) Hematologic complications of human immunodeficiency virus infection and the acquired immunodeficiency syndrome. Med Clin North Am 81, 449-470.

46. Sullivan PS, Hanson DL, Chu SY et al. (1998) Epidemiology of anemia in human immunodeficiency virus (HIV)infected persons: results from the multistate adult and adolescent spectrum of HIV disease surveillance project. Blood 91, 301-308.

47. Kreuzer KA \& Rockstroh JK (1997) Pathogenesis and pathophysiology of anemia in HIV infection. Ann Hematol 75, 179-187.

48. Schilling RF (1991) Anemia of chronic disease: a misnomer. Ann Intern Med 115, 572-573.

49. Fauci AS, Braunwald E, Kasper DL et al. (2008) Harrison's Principles of Internal Medicine, 17th ed. New York: McGraw-Hill.

50. Mehta S, Giovannucci E, Mugusi FM et al. (2010) Vitamin D status of HIV-infected women and its association with HIV disease progression, anemia, and mortality. PLOS ONE 5, e8770.

51. Yetgin S, Ozsoylu S, Ruacan S et al. (1989) Vitamin Ddeficiency rickets and myelofibrosis. J Pediatr 114, 213-217.

52. Kendrick J, Targher G, Smits G et al. (2009) 25-Hydroxyvitamin $\mathrm{D}$ deficiency and inflammation and their association with hemoglobin levels in chronic kidney disease. Am J Nephrol 30, 64-72.

53. Lawson M \& Thomas M (1999) Vitamin D concentrations in Asian children aged 2 years living in England: population survey. BMJ 318, 28.

54. Grindulis H, Scott PH, Belton NR et al. (1986) Combined deficiency of iron and vitamin D in Asian toddlers. Arch Dis Child 61, 843-848.

55. Standing Committee on the Scientific Evaluation of Dietary Reference Intakes, Food and Nutrition Board, Institute of Medicine (2001) Dietary Reference Intakes for Vitamin A, Vitamin K, Arsenic, Boron, Chromium, Copper, Iodine, Iron, Manganese, Molybdenum, Nickel, Silicon, Vanadium, and Zinc. Washington, DC: National Academy Press.

56. Beard J (2006) Iron. In Present Knowledge in Nutrition, 9th ed., pp. 430-444 [BA Bowman and RM Russell, editors]. Washington, DC: International Life Sciences Institute. 